\title{
Tratamiento del hepatocarcinoma
}

\author{
B. Sangro Gómez-Acebo y J. Prieto Valtueña \\ Unidad de Hepatología. Clínica Universitaria de Navarra. Pamplona.
}

Correspondencia: B. Sangro Gómez-Acebo.

Unidad de Hapatología.

Clínica Universitaria de Navarra.

Avda. de Pío XII, 36.

31008 Pamplona

Correo electrónico: bsangro@unav.es

En las últimas dos décadas se han desarrollado formas eficaces de tratamiento del hepatocarcinoma (HCC) cuya aplicación ha contribuido a mejorar el pronóstico de una porción relevante de los pacientes que padecen esta enfermedad (tabla 1). Sin embargo, la elección de la actitud terapéutica adecuada para cada caso individual sigue siendo en ocasiones una tarea complicada, tanto por la heterogeneidad biológica de este tumor como por la complejidad técnica de las opciones terapéuticas y por el hecho de que aparezca habitualmente en pacientes con cirrosis hepática. Esto último, en nuestro entorno geográfico, ocurre en 9 de cada 10 pacientes ${ }^{1}$, por lo que este comentario se limitará a considerar el tratamiento del HCC que aparece en el paciente cirrótico.

La cirugía ocupa un lugar principal en el tratamiento del HCC. En centros con experiencia la resección hepática es un procedimiento seguro, con una mortalidad del $5 \%^{2}$, y permite obtener una supervivencia a 5 años del $30 \%-40 \%$ en la mayoría de las series amplias ${ }^{3,4}$. No obstante, la recidiva es muy frecuente a largo plazo y depende fundamentalmente de dos factores que sólo se conocen tras la intervención, como son la invasión vascular y la, presencia de lesiones satélite ${ }^{5}$. Además, la supervivencia tras la resección no depende sólo de la posible recidiva, sino también de la progresión de la hepatopatía crónica subyacente, por lo que la cirugía de resección debe reservarse para aquellos pacientes sin hipertensión portal aparente y con función hepatocelular próxima a la normal, y no debe realizarse más que en centros con suficiente experiencia ${ }^{6}$. Por otro lado, el trasplante hepático ofrece la mejor supervivencia a largo plazo cuando la elección del paciente es adecuada, lo que no extraña en tanto en cuanto elimina radicalmente el tumor y las frecuentes micrometástasis intrahepáticas, suprime el riesgo de neocarcinogénesis e impide las complicaciones de la cirrosis subyacente. Los factores que determinan el riesgo de recidiva tras el trasplante son la invasión vascular, el tamaño del tumor, el número de nódulos y quizá el grado de diferenciación ${ }^{7}$. Por ello, los criterios actualmente más extendidos limitan la indicación para los pacientes con tumor único de hasta $5 \mathrm{~cm}$, o hasta tres nódulos de no más de $3 \mathrm{~cm}$, y en cualquier caso sin invasión vascular o afectación extrahepática. En estas condiciones la supervivencia de los pacientes cirróticos sometidos a trasplante no difiere según tengan $\mathrm{HCC}$ o no lo tengan ${ }^{8}$, de modo que la indicación por $\mathrm{HCC}$ ha supuesto el $16 \%$ de todas las indicaciones de trasplante en España en el año $2001^{9}$. 
Uno de los avances importantes en el tratamiento del HCC ha sido el desarrollo de diversos procedimientos para la destrucción de tumores utilizando métodos físicos o químicos aplicados con mayor o menor sencillez por vía percutánea. El primero y más extendido es la inyección de sustancias con capacidad necrotizante como el alcohol absoluto. La inyección percutánea de etanol es un procedimiento relativamente sencillo, seguro y barato que se realiza en una o varias sesiones, generalmente bajo control ecográfico. Los resultados en términos de erradicación del tumor dependen del tamaño de la lesión, de forma que se consigue en el $100 \%$ de los tumores de $2 \mathrm{~cm}$ o menos ${ }^{10} \mathrm{y}$ en porcentajes menores de los tumores mayores. Aunque no se dispone de estudios randomizados, los estudios de cohortes han señalado que la supervivencia de los pacientes con tumores de tamaño inferior a $5 \mathrm{~cm}$ y función hepática conservada tratados de esta forma es similar a la de los pacientes sometidos a resección ${ }^{11}$. Una alternativa a la inyección de etanol que se viene extendiendo en los últimos años es la destrucción del tumor mediante la aplicación local de calor, producido por un generador de radiofrecuencia que se acopla a un electrodo de aguja, que a su vez se introduce en el seno del tumor. Con este método se puede lograr la necrosis total en lesiones de hasta 5 $\mathrm{cm}$ y la recidiva in situ es infrecuente si se respetan los límites de tamaño ${ }^{12}$. Sus principales limitaciones son el elevado coste y la ineficacia sobre lesiones adyacentes a vasos sanguíneos en virtud de la disipación del calor producido; su principal virtud es que el tratamiento se completa en una sola sesión en la mayoría de los casos.

Las distintas variantes del procedimiento de embolización arterial se basan en dos características de la mayoría de los nódulos de HCC: su vascularización preferentemente arterial, que permite la necrosis masiva tras la deprivación del flujo arterial, y su capacidad para retener el contraste oleoso Lipiodol ${ }^{\circledR}$, que ha generado la teoría de que la mezcla con fármacos antineoplásicos puede permitir incrementar la disponibilidad de éstos en el tumor, aunque este punto nunca ha sido comprobado experimentalmente. La quimioembolización es el procedimiento más usual y consiste en la inyección en la arteria hepática, o una de sus ramas, de una mezcla de Lipiodol ${ }^{\circledR}$ y algún citostático (habitualmente adriamicina o cisplatino) seguida de la inyección de una sustancia embolizante. Las variaciones en la técnica (cateterización superselectiva de una arteria nutricia o de la hepática común), en el agente embolizante (polvo o esponja de gelatina, polivinilalcohol, embosferas, coils metálicos), en el fármaco antineoplásico o en la frecuencia de repetición del tratamiento complican enormemente el análisis de los datos disponibles en la literatura. Los estudios retrospectivos o de cohortes que comparan las técnicas embolizantes con otros tratamientos, o ninguno, ofrecen una ventaja no despreciable a favor de la embolización ${ }^{13,14}$. Sin embargo, sólo recientemente un análisis sistemático de los ensayos randomizados ha confirmado esta eficacia de forma rotunda ${ }^{15}$. Aun así, la respuesta a la embolización arterial es muy dispar y el pronóstico es peor en los pacientes con mala función hepatocelular, trombosis portal, tumores grandes y cuando la retención de Lipiodol ${ }^{\circledR}$ es escasa ${ }^{16,17}$. Por todo ello, la embolización arterial debe considerarse sólo para aquellos pacientes con buena función hepática y suficiente flujo portal y sin ascitis, y debe realizarse de la forma más selectiva posible, siendo aconsejable utilizar Lipiodol ${ }^{\circledR}$ para poder establecer precozmente el grado de necrosis producida y determinar así la conveniencia de la repetición.

El HCC es muy poco quimiosensible, tanto a los fármacos tradicionales como a los más modernos, y aunque la administración de los citostáticos por vía intraarterial puede incrementar el efecto antitumoral medido por la tasa de respuestas ${ }^{18}$, no parece que 
ayude a prolongar la supervivencia ${ }^{14,19}$. Por su lado, los intentos de manipulación hormonal utilizando tamoxifeno, antiandrógenos o combinaciones de ambos no produce beneficio terapéutico alguno ${ }^{19}$, aunque sigan empleándose como placebo en muchos centros.

Las mejoras en los procedimientos de irradiación, que permiten concentrar las dosis lesivas en el área tumoral, han permitido en los últimos años retomar la radioterapia externa como método de tratamiento en esta enfermedad. Con la aplicación de dosis de hasta 70 Gy se obtiene un efecto antitumoral considerable ${ }^{20}$, que puede contribuir a lograr la resecabilidad en lesiones únicas de gran tamaño. Un efecto paliativo interesante puede conseguirse también con técnicas de irradiación selectiva, como la inyección intraarterial hepática de microesferas cargadas de ${ }^{90} \mathrm{Y}^{21} \mathrm{o}$ Lipiodol ${ }^{\circledR}$ marcado con ${ }^{131} \mathrm{I}^{22}$.

Con esta variedad de elementos terapéuticos pueden surgir muchas dudas a la hora de elegir el mejor tratamiento para cada paciente concreto. Cuando la enfermedad se detecta en estadio precoz, y por tanto potencialmente curable, la primera opción que debe valorarse es la del trasplante hepático, puesto que ofrece la mejor supervivencia a largo plazo. En la decisión de indicar el trasplante debe tenerse en cuenta la disponibilidad de órganos y las contraindicaciones a la intervención. Si el trasplante hepático no fuera indicado, el grado de deterioro de la hepatopatía de base marca la siguiente decisión. Los pacientes con grave deterioro (estadio C de Child-Pugh) deben recibir únicamente tratamiento sintomático. En los demás casos, para tomar la decisión de someterlos a resección quirúrgica o destrucción percutánea debe tenerse en cuenta la disponibilidad y experiencia con uno y otro método en cada centro, debiendo reservarse en cualquier caso la resección para aquellos pacientes con hepatopatía mínimamente avanzada, y en centros con experiencia en este tipo de cirugía. En la situación del paciente con enfermedad limitada al hígado, pero no abordable por métodos radicales, la embolización arterial puede mejorar la supervivencia e incluso conseguir el rescate quirúrgico o percutáneo en algunos casos, pero debe evitarse si existe compromiso del flujo portal o mala función hepática, sobre todo si resulta precisa una embolización no selectiva. En los casos en que los procedimientos antedichos no sean aplicables por uno u otro motivo es más que aconsejable que se ofrezca a los pacientes la posibilidad de participar en ensayos clínicos que permitan establecer la utilidad de nuevos fármacos y procedimientos o combinaciones de los ya existentes.

\section{BIBLIOGRAFÍA}

1. Caivet X, Bruix J, Bru C, Ginés P, Vilana R, Sole M, et al. Natural history of hepatocellular carcinoma in Spain. Five year's experience in 249 cases. Hepatol 1990;10:311-7.

2. Fong Y, Sun RL, Jarnagin W, Blumgart LH. An analysis of 412 cases of hepatocellular carcinoma at a Western center [discussion 799-800]. Ann Surg 1999;229:790-9;

3. Lau H, Fan ST, Ng IO, Wong J. Long term prognosis after hepatectomy for hepatocellular carcinoma: a survival analysis of 204 consecutive patients. Cancer 1998;83:2302-11. 
4. Mazziotti A, Grazi GL, Cavallari A. Surgical treatment of hepatocellular carcinoma on cirrhosis: a Western experience. Hepato-Gastroenterology 1998;45(Suppl 3):1281-7.

5. Poon RT, Fan ST, Ng IO, Lo CM, Liu CL, Wong J. Different risk factors and prognosis for early and late intrahepatic recurrence after resection of hepatocellular carcinoma. Cancer 2000;89:500-7.

6. Llovet JM, Fuster J, Bruix J. Intention-to-treat analysis of surgical treatment for early hepatocellular carcinoma: resection versus transplantation. Hepatology (Baltimore) 1999;30:1434-40.

7. Klintmalm GB. Liver transplantation for hepatocellular carcinoma: a registry report of the impact of tumor characteristics on outcome. Ann Surg 1998;228:479-90.

8. Herrero JI, Sangro B, Quiroga J, Pardo F, Herraiz M, Cienfuegos JA, et al. Influence of tumor characteristics on the outcome of liver transplantation among patients with liver cirrhosis and hepatocellular carcinoma. Liver Transpl 2001;7:631-6.

9. Trasplante hepático. Revista Española de Trasplantes 2002;11:58-78.

10. Vilana R, Bruix J, Bru C, Ayuso C, Sole M, Rodes J. Tumor size determines the efficacy of percutaneous ethanol injection for the treatment of small hepatocellular carcinoma. Hepatology 1992;16:353-7.

11. Livraghi T, Bolondi L, Buscarini L, Cottone M, Mazziotti A, Morabito, et al. No treatment, resection and ethanol injection in hepatocellular carcinoma: a retrospectiva analysis of survival in 391 patients with cirrhosis. Italian Cooperative HCC Study Group. J Hepatol 1995;22:522-6.

12. Livraghi T, Goldberg SN, Lazzaroni S, Meloni F, Solbiati L, Gazelle GS. Small hepatocellular carcinoma: treatment with radio-frequency ablation versus ethanol injection. Radiology 1999;210:655-61.

13. Bronowicki JP, Vetter D, Dumas F, Boudjema K, Bader R, Weiss AM, et al. Transcatheter oily chemoembolization for hepatocellular carcinoma. A 4-year study of 127 French patients. Cancer 1994;74:16-24.

14. Sangro B, Herraiz M, Martínez-González MA, Bilbao I, Herrero I, Beloqui O, et al. Prognosis of hepatocellular carcinoma in relation to treatment: a multivariate analysis of 178 patients from a single European institution [published erratum appears in Surgery 1998;124(6):1087]. Surgery 1998;124:575-83.

15. Llovet JM, Bruix J. Systematic review of randomized trials for unresectable hepatocellular carcinoma: chemoembolization improves survival. Hepatology 2003;37:429-42.

16. Figueras J, Jaurrieta E, Valls C, Benasco C, Rafecas A, Xiol X, et al. Survival after liver transplantation in cirrhotic patients with and without hepatocellular carcinoma: a comparative study. Hepatology 1997;25: 1485-9.

17. Hatanaka Y, Yamashita Y, Takahashi M, Koga Y, Saito R, Nakashima K, et al. Unresectable hepatocellular carcinoma: analysis of prognostic factors in transcatheter management. Radiology 1995;195:747-5z.

18. Sangro B, Ríos R, Bilbao I, Beloqui O, Herrero JI, Quiroga J, et al. Efficacy and toxicity of intra-arterial cisplatin and etoposide for advanced hepatocellular carcinoma. Oncology 2002;62:293-8.

19. Simonetti RG, Liberati A, Angiolini C, Pagliaro L. Treatment of hepatocellular carcinoma: a systematic review of randomized controlled trials. Ann Oncol 1997;8:117-36. 
20. Cheng SH, Lin YM, Chuang VP, Yang PS, Cheng JC, Huang AT, et al. A pilot study of three-dimensional conformal radiotherapy in unresectable hepatocellular carcinoma. J Gastroenterol Hepatol 1999;14:1025-33.

21. Salem R, Thurston KG, Carr BI, Goin JE, Geschwind JF. Yttriurn-90 microspheres: radiation therapy for unresectable liver cancer. J Vasc Interv Radiol 2002;13:S223-9.

22. Risse JH, Grunwald F, Kersjes W, Strunk H, Caselmann WH, Palmado H, et al. Intraarterial HCC therapy with I-131-Lipiodol. Cancer Biother Radiopharm 2000;15:65-70. 


\begin{tabular}{|c|c|c|}
\hline \multicolumn{3}{|c|}{$\begin{array}{l}\text { Tabla 1. Procedimientos terapéuticos con efecto antitumoral conocido } \\
\text { frente al hepatocarcinoma }\end{array}$} \\
\hline \multirow{2}{*}{ Procedimiento } & \multicolumn{2}{|c|}{ Población diana } \\
\hline & Características del tumor & Función hepática e HTP \\
\hline \multirow[t]{2}{*}{ Trasplante } & $\begin{array}{l}\text { Tumor único de }<5 \mathrm{~cm} \mathrm{o} \\
\text { hasta } 3 \text { nódulos de } 3 \mathrm{~cm}\end{array}$ & Indiferente \\
\hline & $\begin{array}{l}\text { No invasión vascular ni } \\
\text { enfermedad extrahepática }\end{array}$ & \\
\hline Resección & $\begin{array}{l}\text { No invasión vascular ni } \\
\text { enfermedad extrahepática }\end{array}$ & Child A sin HTP \\
\hline \multirow[t]{2}{*}{ IPE / RF } & Tumores de hasta $5 \mathrm{~cm}$ & Child A o B \\
\hline & $\begin{array}{l}\text { No invasión vascular ni } \\
\text { enfermedad extrahepática }\end{array}$ & \\
\hline Embolización arterial & $\begin{array}{l}\text { No invasión vascular ni } \\
\text { enfermedad extrahepática }\end{array}$ & Child A o B \\
\hline Radioterapia & Por establecer & Child A o B \\
\hline \multicolumn{3}{|c|}{$\begin{array}{l}\text { HTP: hipertensión portal } \\
\text { IPE: inyección percutánea de etanol } \\
\text { RF: ablación por radiofrecuencia }\end{array}$} \\
\hline
\end{tabular}

